\title{
(-)-Epicatechin protects against myocardial ischemia-induced cardiac injury via activation of the PTEN/PI3K/AKT pathway
}

\author{
JIA-WEN LI ${ }^{1}$, XIAO-YUN WANG ${ }^{2}$, XIN ZHANG $^{1}$, LEI GAO $^{1}$, LI-FENG WANG $^{2}$ and XIN-HUA YIN ${ }^{1}$ \\ ${ }^{1}$ Department of Cardiology, First Affiliated Hospital, Harbin Medical University; ${ }^{2}$ Department of Cardiology, \\ Fourth Affiliated Hospital, Harbin Medical University, Harbin, Heilongjiang 150001, P.R. China
}

Received October 20, 2017; Accepted March 8, 2018

DOI: $10.3892 / \mathrm{mmr} .2018 .8870$

\begin{abstract}
Flavonol (-)-epicatechin (EPI) is primarily contained in green tea (Camellia sinensis) and cocoa beans (Theobroma cacao), and has been demonstrated to be beneficial for the health of the cardiovascular system. However, the effect and the underlying mechanism of EPI on myocardial ischemia induced cardiac injury has not yet been determined. Therefore, the present study aimed to detect whether EPI inhibited myocardial ischemia injury. An in vivo mouse myocardial ischemia model was induced by the ligation of left descending coronary artery for 7 days. EPI ( $1 \mathrm{mg} / \mathrm{kg} / \mathrm{day})$ was administrated 10 days prior to myocardial ischemia operation. The in vitro mouse myocardial ischemia model was induced by cultivating neonatal mouse cardiomyocytes under anoxia condition for $12 \mathrm{~h}$. Cardiomyocytes were treated with EPI $(5 \mu \mathrm{M})$ for $1 \mathrm{~h}$ and then incubated under anoxia conditions. Mouse hearts and cultured cardiomyocytes were used for hematoxylin-eosin, masson, ultrasonography, terminal dUTP nick end-labeling, immunofluorescence, western blotting and MTT assays. Results revealed that myocardial ischemia-induced mouse cardiac injury was significantly inhibited by EPI, as evidenced by decreased myocardial apoptosis, cardiac fibrosis and myocardial hypertrophy and improved cardiac function. In addition, it was confirmed that EPI serves a protective effect against myocardial ischemia via the phosphatase and tensin homolog (PTEN)/phosphoinositide 3-kinase (PI3K)/protein kinase B (AKT) signaling pathway, which was reversed by the PI3K inhibitor, LY294002. The protective role of EPI in myocardial apoptosis was further confirmed on mouse cardiomyocytes following anoxia treatment in vitro. In conclusion, the data suggested that EPI protects against myocardial ischemia induced cardiac injury through the PTEN/PI3K/AKT signaling pathway in vivo and
\end{abstract}

Correspondence to: Professor Xin-Hua Yin, Department of Cardiology, First Affiliated Hospital, Harbin Medical University, 23 Youzheng Street, Harbin, Heilongjiang 150001, P.R. China E-mail: yinxinhua5063@163.com

Key words: epicatechin, myocardial ischemia, myocardial injury, apoptosis, phosphatase and tensin homolog, protein kinase B in vitro, which may provide clinical therapeutic approaches and targets for cardiac ischemia injury.

\section{Introduction}

Myocardial ischemia is a direct result of coronary artery stenosis or occlusion, which is characterized by high morbidity and mortality worldwide (1). Myocardial ischemia, especially acute myocardial ischemia, has been estimated by the World Health Organization to be the primary cause of death in the world by the year 2020 (2). Except sudden death, myocardial ischemia causes cardiomyocyte hypertrophy, cardiomyocyte apoptosis, cardiac fibrosis and cardiac dysfunction (3-5). Therefore, interventions and mechanisms involved in myocardial ischemia have been explored. For example, rapid reperfusion is an active treatment for acute myocardial ischemia, which attenuates myocardial infarction and relieves cardiac dysfunction (6). However, there remains a requirement for more active and more convenient treatments for myocardial ischemia.

(-)-Epicatechin (EPI) belongs to the group of flavanols, which is primarily contained in flavonoid-rich foods, such as green tea and dark chocolate (7). It is believed that EPI contributes to the beneficial effects of flavonoid-rich foods on the cardiovascular system. Research has indicated that EPI protects hearts from myocardial ischemia reperfusion injury, cardiac hypertrophy, oxidative stress injury and coronary occlusion (8-11). Catechin, a conformational isomer of EPI, was demonstrated to attenuate chronic ventricular remodeling induced by myocardial ischemia in rats (12). Epigallocatechin gallate, an analogue of EPI, also inhibits telomere attrition induced cardiomyocyte apoptosis (11). However, it is still unclear whether EPI has an effect on myocardial ischemia in vivo and cardiomyocyte apoptosis in vitro.

Dysregulation of numerous signaling pathways has been demonstrated to be involved in myocardial ischemia induced cardiac injury, especially the phosphatidylinositol 3-kinase $(\mathrm{PI} 3 \mathrm{~K}) /$ protein kinase B $(\mathrm{AKT})$ pathway $(13,14)$. This pathway is an intracellular signaling pathway, which serves a role in modulating cell proliferation, differentiation, autophagy and apoptosis under physiological and pathological conditions (15). The activation of the PI3K/AKT pathway interacts with complexed downstream target proteins including B-cell lymphoma-2 (Bcl-2) and Bcl-2-associated X protein (Bax) 
to promote cell survival, which is negatively regulated by phosphatase and tensin homolog (PTEN) (16). The PI3K/AKT pathway was shown to be a promising therapy target for myocardial ischemia. For example, Ke et al (13) revealed that the activation of the PI3K/AKT pathway protects against isoproterenol-induced myocardial ischemic injury. EPI was demonstrated to induce physiological cardiac growth by activating the PI3K/AKT pathway (17). The aforementioned results suggested that EPI may exhibit an anti-myocardial ischemia function via activation of the PI3K/AKT pathway.

Therefore, the present study aimed to investigate the effect of EPI on mouse myocardial ischemic injury induced by coronary occlusion in vivo and by anoxia cultivation in vitro. Furthermore, the present study also investigated the mechanism underlying cardiac protection of EPI, which promotes the activation of the PTEN/PI3K/AKT signaling pathway to inhibit apoptosis of cardiomyocytes.

\section{Materials and methods}

Animals. A total of 24 healthy male C57BL/6 mice at the age of eight weeks, $25-30 \mathrm{~g}$ in weight, were obtained from the experimental Animal Center of the Harbin Medical University (Harbin, China). The mice were randomly divided into the sham group, myocardial ischemia (MI) group, MI+EPI group and MI+EPI+LY294002 group, and n=6 in each group. Subsequently, according to the different groups, the mice were subjected to a sham or MI operation and the mice that underwent MI surgery were administered EPI or EPI+LY294002. The animals were kept under standard animal room conditions (atmosphere $0.03 \% \mathrm{CO}_{2}$; temperature $21 \pm 1^{\circ} \mathrm{C}$; humidity $55-60 \%$; 12-h light-dark cycle) with laboratory pellet food and autoclaved water freely available. All experimental procedures were performed in accordance with the Guidelines for the Care and Use of Laboratory Animals set by the US National Institutes of Health (Bethesda, MD, USA), which was approved by the Institutional Animal Care and Use Committee of the Harbin Medical University.

Myocardial ischemia model establishment and drug treatments in vivo. To induce myocardial ischemia, mice were anesthetized with Avertin $(160 \mathrm{mg} / \mathrm{kg}$; intraperitoneal injection; Sigma-Aldrich, Merck KGaA, Darmstadt, Germany) and their chests were opened to expose the hearts. The left descending coronary artery was ligated with a 7/0 nylon suture at $2 \mathrm{~mm}$ below the border between the left atrium and ventricle to induce myocardial ischemia. Ischemia was confirmed by visual observation (cyanosis) and by observing ST segment elevation and QRS widen on electrocardiogram. The sham group mice underwent the same experimental procedures as the MI group but without ligation of left descending coronary artery. Prior to the myocardial ischemia operation, the mice in the MI+EPI (cat. no. 68097; Sigma-Aldrich; Merck KGaA, Darmstadt, Germany) and MI+EPI+LY294002 (cat. no. L9908; Sigma-Aldrich, Merck KGaA) groups were treated with $1 \mathrm{mg}$ EPI per kg body weight by oral gavage every day for 10 days. Additionally, the mice in the MI+EPI+LY294002 group were treated with LY294002 at $10 \mathrm{mg} / \mathrm{kg}$ body weight by intraperitoneal injection $30 \mathrm{~min}$ before treatment of EPI every day for 10 days. The mice in the sham group were administered with an equal dosage of saline. All mice were sacrificed at day 7 after the myocardial ischemia operation.

Treatment of cardiomyocytes. Cardiomyocytes were isolated from neonatal C57BL/6 mice (aged 1-2 days, weight 1-3 g, a random mix of male or female; purchased from the experimental Animal Center of Harbin Medical University) as previously described, from mice that were sacrificed following purchase (18). Briefly, the heart was digested by pancreatin (cat no. C0201; Beyotime Institute of Biotechnology, Haimen, China) and maintained in Dulbecco's modified Eagle's medium (DMEM; cat no. 11965084; Gibco; Thermo Fisher Scientific, Inc., Waltham, MA, USA) supplemented with $100 \mathrm{U} / \mathrm{ml}$ penicillin and $10 \%$ fetal bovine serum (FBS; cat no. 04-001-1A; Biological Industries, Kibbutz Beit Haemek, Israel) (18). Cardiomyocytes were purified by differential adherence and $0.1 \mathrm{mM}$ 5-bromo-2-deoxyuridine (cat no. B23151; Thermo Fisher Scientific, Inc.) was added to suppress cardiac fibroblasts for $48 \mathrm{~h}$ in a $5 \% \mathrm{CO}_{2}$ and $37^{\circ} \mathrm{C}$ humidified atmosphere. Then, as the anoxia (ANO) group, ANO+EPI group or ANO+EPI+LY group, the purified cardiomyocytes were treated with an equal amount of DMEM or EPI $(5 \mu \mathrm{M})$ or EPI $(5 \mu \mathrm{M})+\mathrm{LY} 294002(20 \mu \mathrm{M})$ separately in a $5 \% \mathrm{CO}_{2}$ and $37^{\circ} \mathrm{C}$ humidified atmosphere for $1 \mathrm{~h}$ and then incubated under anoxia condition at $37^{\circ} \mathrm{C}$ for $12 \mathrm{~h}$. As the control group, the purified cells were treated with an equal amount of DMEM in a $5 \% \mathrm{CO}_{2}$ and $37^{\circ} \mathrm{C}$ humidified atmosphere for $13 \mathrm{~h}$.

Echocardiograph. Transthoracic echocardiography was performed to measure alterations to the left ventricular function using a Vevo2100 system (VisualSonics, Inc., Toronto, ON, Canada) equipped with a 10-MH2 phased-array transducer with the M-mode recordings. Functional parameters were evaluated and analyzed including heart rate (HR), left ventricular end-diastolic dimensions (LVEDd), left ventricular end-systolic dimensions (LVESd), left ventricular ejection fraction (EF) and fractional shortening (FS).

Histological analysis. Hearts were collected and fixed with $4 \%$ paraformaldehyde at $4^{\circ} \mathrm{C}$ for $48 \mathrm{~h}$, embedded in paraffin, sliced into $5-\mu \mathrm{m}$ sections and subjected to standard hematoxylin and eosin $(\mathrm{H} \& \mathrm{E})$ staining and Masson staining at room temperature for $4 \mathrm{~h}$. The sections were visualized using a light microscope (80i; Nikon Corporation, Tokyo, Japan). A total of 10 fields of view each were analyzed. Image J software (version 1.46r; National Institutes of Health, USA) was used for quantitative analysis of the $\%$ area of fibrosis.

Cell viability assay. Cell viability was measured by the mitochondrial dependent reduction of MTT reagent (cat no. C0009; Beyotime Biotechnology, Shanghai, China), as previously described (19). Neonatal mouse cardiomyocytes (NMCMs) were plated onto a 96-well plate and treated with an equal amount of DMEM or EPI $(5 \mu \mathrm{M})$ or EPI $(5 \mu \mathrm{M})+\mathrm{LY} 294002$ $(20 \mu \mathrm{M})$ in a $5 \% \mathrm{CO}_{2}$ and $37^{\circ} \mathrm{C}$ humidified atmosphere for $1 \mathrm{~h}$ and then incubated under anoxia condition at $37^{\circ} \mathrm{C}$ for $12 \mathrm{~h}$. As control group, NMCMs plated onto a 96 -well plate treated with an equal amount of DMEM in a $5 \% \mathrm{CO}_{2}$ and $37^{\circ} \mathrm{C}$ humidified atmosphere for $13 \mathrm{~h}$, as designated. A total of $20 \mu \mathrm{l} \mathrm{MTT}$ solution (Sigma-Aldrich, Merck KGaA) and $180 \mu \mathrm{l}$ DMEM was 

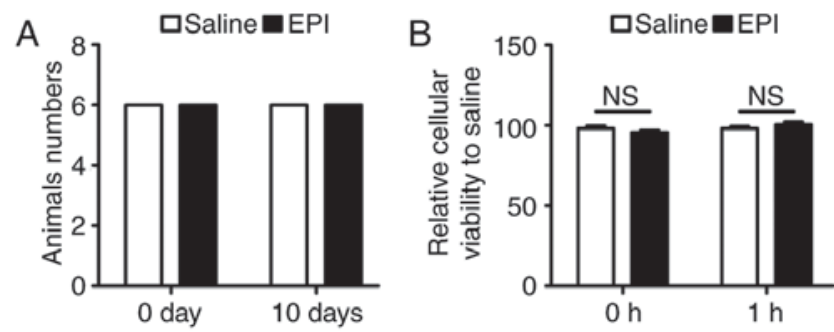

Figure 1. Safety dosage of EPI for treating myocardial ischemia in vivo and in vitro. (A) Overall survival of mice administrated with EPI or saline at 0 or 10 days. (B) Relative viability of cardiomyocytes treated with saline or EPI for $1 \mathrm{~h}$. Data are expressed as the mean \pm standard error $(n=6)$. EPI, (-)-epicatechin; NS, not significant.

added into each well and the plates were incubated at $37^{\circ} \mathrm{C}$ for $4 \mathrm{~h}$. Then, the medium of each well was carefully removed and $200 \mu 1$ DMSO was added into each well to dissolve formazan. The absorbance values were read at a wavelength of $490 \mathrm{~nm}$ using a microplate reader (Tecan Group, Ltd., Mannedorf, Switzerland).

Western blot analysis. For Western blot analysis, protein samples extracted from the left ventricles of mice or cultured NMCMs were used. Briefly, protein was lysed with radioimmunoprecipitation assay lysis buffer containing a complete protease inhibitor cocktail (cat. no. 4693116001; Roche Molecular Diagnostics, Pleasanton, CA, USA). The protein concentration was determined by BCA Protein Assay kit (cat no. P0012S; Beyotime Institute of Biotechnology, Shanghai, China). A total of $10 \mu \mathrm{g}$ protein was separated by $12 \%$ SDS-PAGE and transferred onto polyvinylidene difluoride membranes. The blots were probed with primary antibodies overnight at $4^{\circ} \mathrm{C}$. The primary antibodies included Bcl-2 (1:1,000; cat no. 3498), Bax (1:1,000; cat no. 14796), caspase-3 (1:500; cat no. 9665), cleaved caspase-3 (1:500; cat no. 9661), PTEN (1:1,000; cat no. 9559; all Cell Signaling Technology, Inc., Danvers, MA, USA), total AKT (T-AKT; 1:200; cat no. sc-8312; Santa Cruz Biotechnology, Inc., Dallas TX, USA), phosphorylated-AKT (p-AKT; 1:1,000; cat no. 13038) and GAPDH (1:1,000; cat no. 2118; both Cell Signaling Technology, Inc.). After washing, the membranes were incubated with a horse radish peroxidase-conjugated goat-anti-rabbit immunoglobulin G (1:1,000; cat no. A0208; Beyotime Institute of Biotechnology) for $1 \mathrm{~h}$ at room temperature. The signal was detected by an Enhanced Chemiluminescence reagent (cat no. 29050; Engreen Biosystem New Zealand Ltd., Auckland, New Zealand), quantified by using Odyssey Infrared Imaging System (LI-COR Biosciences, Lincoln, NE, USA) and analyzed by Odyssey Infrared Imaging System matched application software (version 3.0.16; LI-COR Biosciences, Lincoln, NE, USA).

Terminal deoxynucleotidyl transferase-mediated dUTP nick-end labeling (TUNEL) assay. Apoptosis was assessed by a TUNEL assay (Roche Molecular Diagnostics), as previously described (20). Then, 6-mm frozen sections or cells were also co-stained with DAPI (1:1,000; cat no. D3571; Invitrogen; Thermo Fisher Scientific, Inc.) at room temperature for $5 \mathrm{mins}$ to visualize nuclei, and mouse monoclonal anti-sarcomeric $\alpha$-actinin antibody (1:1,000; cat no. A7811; Sigma-Aldrich; Merck KGaA) at $4^{\circ} \mathrm{C}$ overnight. Then the sections or cells were incubated with goat-anti-mouse IgG-Alexa Fluor 594 at $37^{\circ} \mathrm{C}$ for 2 h (1:300; cat no. A-11032; Invitrogen; Thermo Fisher Scientific, Inc.). The average of TUNEL-positive cell ratio in at least five representative microscopic fields was calculated with ZEN2012 (version 1.1.2.0; Carl Zeiss AG, Oberkochen, Germany) to compare the apoptosis ratio within different groups.

Statistical analysis. All data was expressed as the mean \pm standard error. GraphPad Prism version 5.0 software (GraphPad Software, Inc., La Jolla, CA, USA) was used for statistical analysis. For two group comparisons, an unpaired Student t-test was used. For multiple group comparisons, a one-way analysis of variance with Bonferroni post-test was used for comparisons between selected two groups as well as Dunnett post-test for comparisons among all other treatment groups to the corresponding control. $\mathrm{P}<0.05$ was considered to indicate a statistically significant difference.

\section{Results}

The safety dosage of EPI for treating myocardial ischemia in vivo and in vitro. The present study confirmed the appropriate dosage of EPI. In a previously published study, EPI was administered at $1 \mathrm{mg} / \mathrm{kg} /$ day via oral gavage for 10 days (21) to maintain a sufficient drug level. Therefore, the present study administered EPI ( $1 \mathrm{mg} / \mathrm{kg}$ daily) to $25-30 \mathrm{~g}$ male C57BL/6 mice for 10 days before left descending coronary artery ligation for in vivo experiments. By paying monitoring the overall survival of the mice, it was confirmed that the drug dosage (1 mg/kg daily) was not lethal (Fig. 1A). Additionally, the eating behavior of the mice was observed daily and documented the weight of them over the 10 days. However, there was no significant difference between the two group and therefore it was concluded that the dose was safe (data not shown). According to previous studies for in vitro experiments (21-23), the present study pretreated NMCMs with $5 \mu \mathrm{M}$ EPI for $1 \mathrm{~h}$ in $5 \% \mathrm{CO}_{2}$ and $37^{\circ} \mathrm{C}$ and then incubated cells under anoxia conditions for $12 \mathrm{~h}$. The possible toxicity effect of EPI on NMCMs was determined by an MTT assay. As shown in Fig. 1B, the dosage of EPI was safe for cardiomyocytes.

EPI attenuates myocardial ischemia-induced cardiac fibrosis and apoptosis which is weakened by the specific PI3K inhibitor, LY294002. C57BL/6 mice underwent left descending coronary artery occlusion operation to induce myocardial ischemic, or sham operation for negative control. $\mathrm{H} \& \mathrm{E}$ and Masson staining analysis demonstrated that the areas of cardiomyocytes and cardiac fibrosis of the MI group mice were significantly increased 1 week following MI operation, when compared with the hearts of the sham group mice. EPI treatment suppressed MI-induced cardiac hypertrophy and fibrosis, as evidenced by decreased areas of cardiomyocytes and cardiac fibrosis in the MI+EPI group mice when compared with the MI group (Fig. 2A). TUNEL assay revealed that cardiomyocyte apoptosis increased in the MI group when compared with the sham group, which was 
A

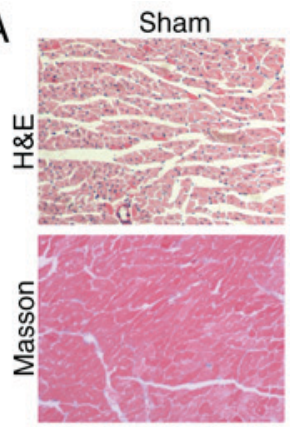

B
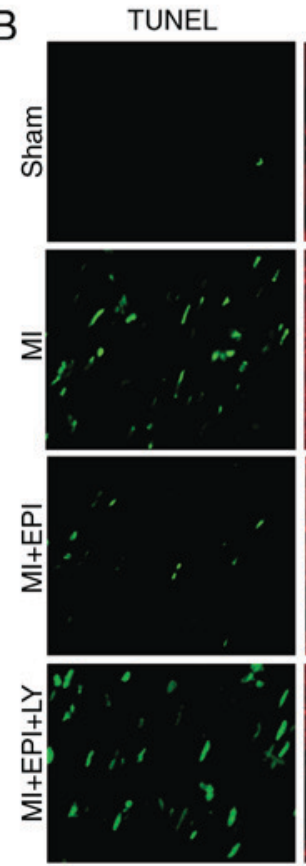

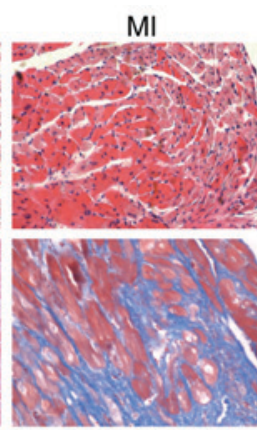

$\alpha$-actinin
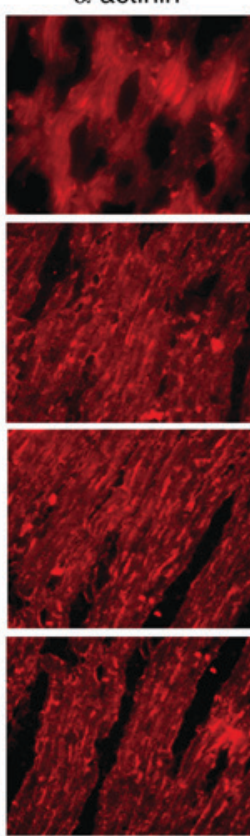

$\mathrm{Ml}+\mathrm{EPI}$

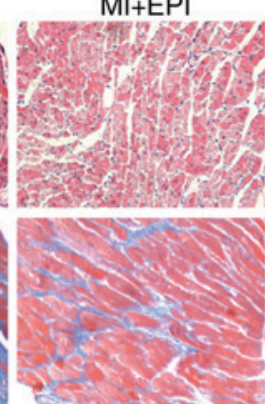

DAPI
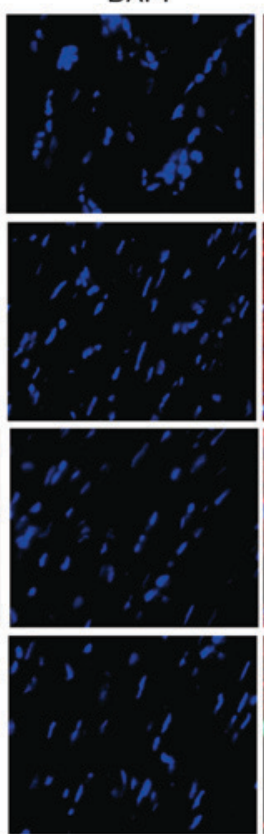

$\mathrm{MI}+\mathrm{EPI}+\mathrm{LY}$

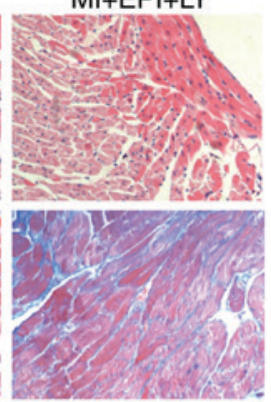

Merge

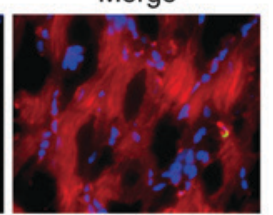

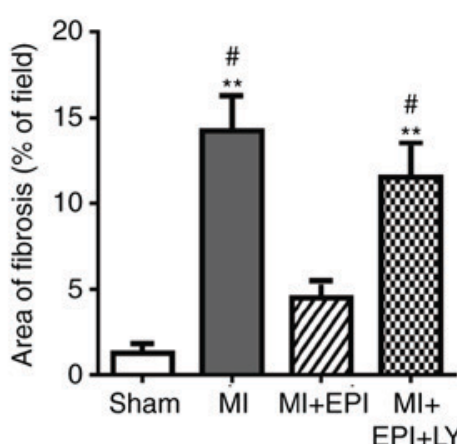

$\mathrm{EPI}+\mathrm{LY}$

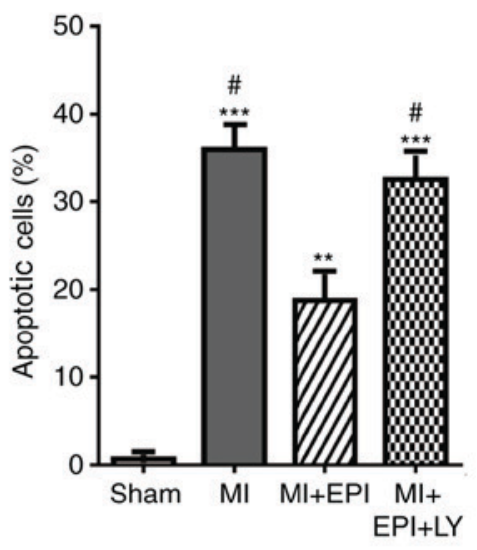

Figure 2. PI3K specific inhibitor LY294002 abolishes the effect of EPI against ischemia-induced mouse cardiac hypertrophy, fibrosis and apoptosis (A) Microscopic examination of histological sections detected by hematoxylin and eosin and Masson staining (magnification, x200) of mouse hearts from the sham group, MI group, MI+EPI group and MI+EPI+LY294002 group. EPI suppressed the increase of the areas of cardiomyocyte and fibers induced by myocardial ischemia operation, which were counteracted by LY294002. (B) Terminal deoxynucleotidyl transferase-mediated dUTP nick-end labeling staining of mouse hearts from the sham group, MI group, MI+EPI group and MI+EPI+LY294002 group. EPI suppressed cellular apoptosis induced by MI operation, which was abolished by LY294002. Data are expressed as the mean \pm standard error $(\mathrm{n}=6) .{ }^{* *} \mathrm{P}<0.01$ and ${ }^{* * *} \mathrm{P}<0.001$ vs. sham. ${ }^{*} \mathrm{P}<0.05$ vs. MI+EPI. MI, myocardial ischemia; EPI, (-)-epicatechin; LY, LY294002. AKT, protein kinase B; EPI, (-)-epicatechin; MI, myocardial injury.

significantly inhibited by EPI administration in the MI+EPI group mice (Fig. 2B). Considering the crucial role of EPI in myocardial ischemia, the underlying molecular mechanism through which EPI exerts its protective effect was further investigated. The PI3K/AKT signaling pathway mediates cell apoptosis, which may be a potential treatment target for ameliorating myocardial ischemia. Therefore, this study used LY294002, a specific PI3K inhibitor, to confirm whether EPI inhibits the myocardial ischemia-induced cardiac injury through the PI3K/AKT pathway. The protective effect of EPI for myocardial ischemia was abolished by LY294002 (Fig. 2A and B). Therefore, these results illustrated that the inhibition of the PI3K/AKT pathway offsets the cardiac protective effect of EPI.

The EPI-mediated improvement of cardiac left ventricular functions is reduced by the PI3k inhibitor LY294002. The present study used two-dimensional echocardiography and $\mathrm{M}$-mode tracings to examine left ventricular functions at day 7 of myocardial ischemic surgery. The left ventricular wall thickness was increased in MI group, which was suppressed by EPI. The effect of EPI on left ventricular wall thickness was attenuated by LY294002 (Fig. 3A). HR, LVEDd, LVESd, EF and fractional shortening FS were detected by transthoracic echocardiography. There was no significant difference of HR between any group (Fig. 3B). EPI significantly inhibited the increase of LVEDd and LVESd induced by the myocardial ischemic operation (Fig. 3C, D). In addition, EPI administration increased FS and EF levels in the MI+EPI group compared with the MI group (Fig. 3E and F). As shown in Fig. 3C and D, LVEDd and the LVESd in the MI+LY294002+EPI group were significantly increased when compared with EPI-treated myocardial ischemic mice. FS and EF were decreased in the MI+LY294002+EPI group after co-treatment with EPI and LY294002 (Fig. 3E, F), compared with the MI+EPI group. These results demonstrated that EPI protects left ventricular function from myocardial ischemic injury via the AKT pathway. 
A

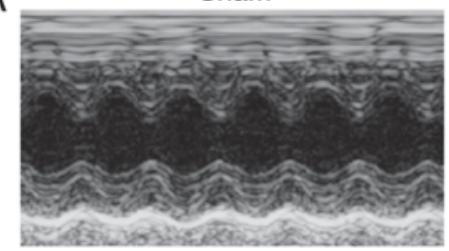

$\mathrm{Ml}+\mathrm{EPI}$

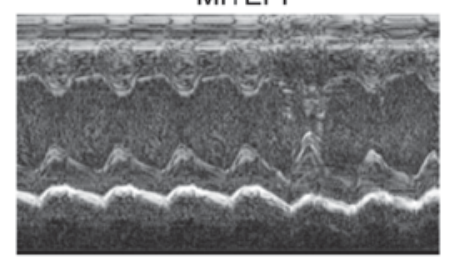

$\mathrm{MI}$

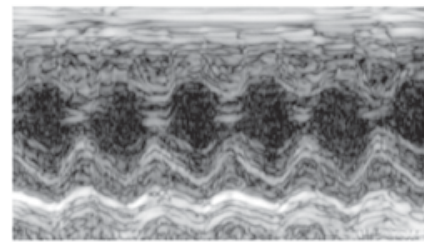

$\mathrm{Ml}+\mathrm{EPI}+\mathrm{LY}$

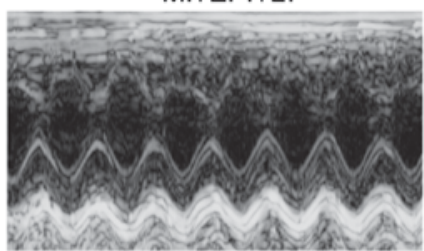

B

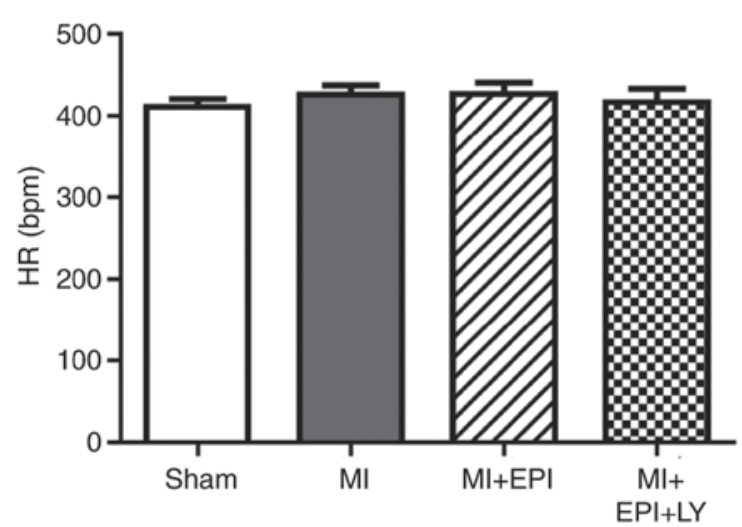

C

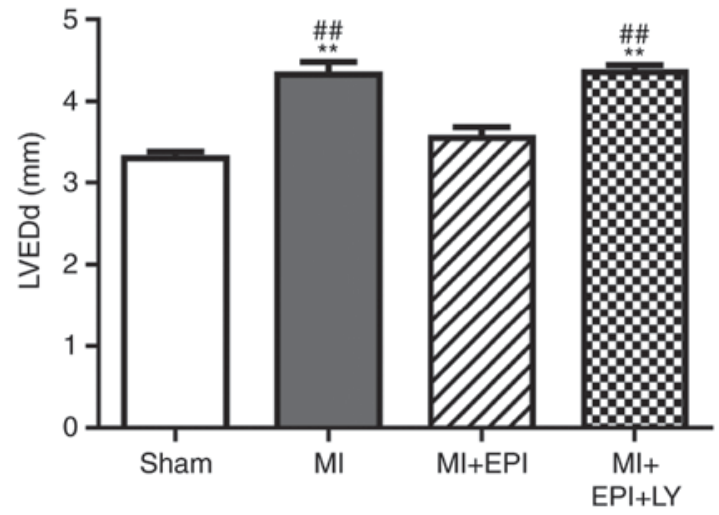

E

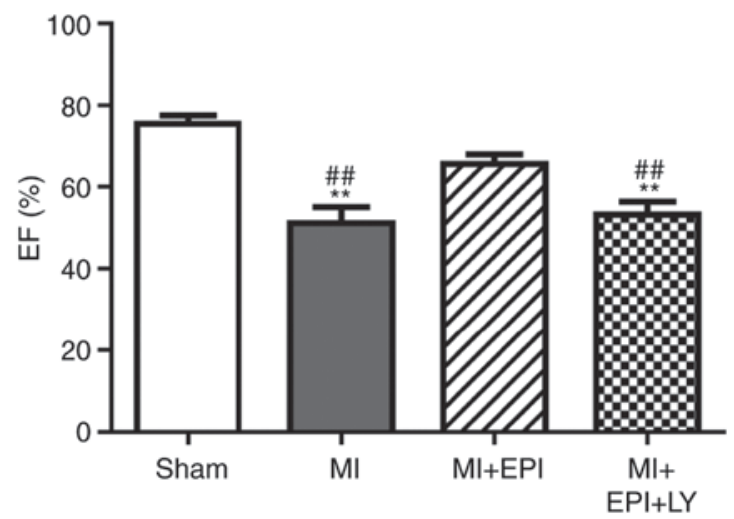

D

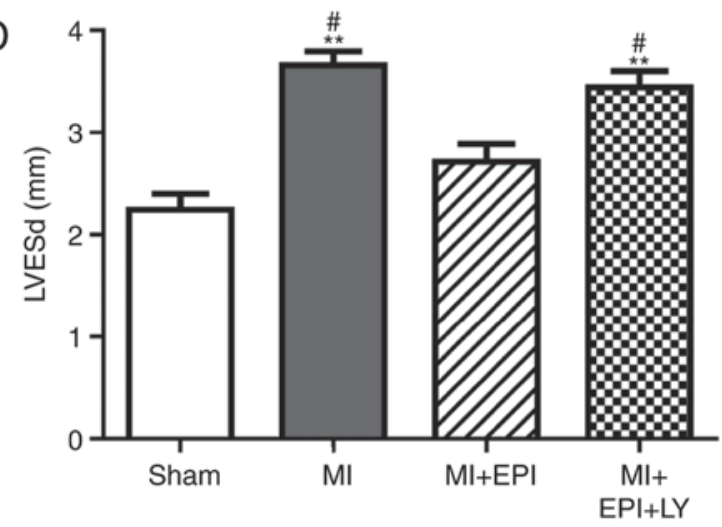

$\mathrm{F}$

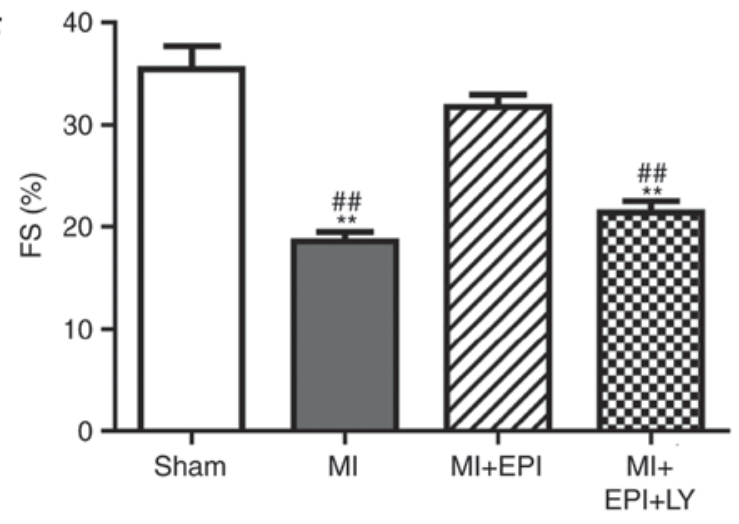

Figure 3. Improvement effect of EPI on cardiac function is counteracted by LY294002. Mice were randomly selected from each group (sham, MI, MI+EPI and MI+EPI+LY294002) and echocardiography was performed. (A) Representative M-mode echocardiographs of the left ventricular wall thickness from the four groups of mice. Each representative image illustrates the continuous changes of left ventricular wall thickness during six to seven mouse cardiac cycles. (B) HR, (C) LVEDd, (D) LVESd, (E) EF and (F) FS. Data are expressed as the mean \pm standard error $(\mathrm{n}=6)$. ${ }^{* *} \mathrm{P}<0.01 \mathrm{vs}$. sham; ${ }^{\#} \mathrm{P}<0.05$ and ${ }^{\# \#} \mathrm{P}<0.01$ vs. MI+EPI. MI, myocardial ischemia; EPI, (-)-epicatechin; LY, LY294002; HR, heart rate; LVEDd, left ventricular end-diastolic dimensions; LVESd, left ventricular end-systolic dimensions; EF, ejection fraction; FS, fractional shortening.

EPI inhibits myocardial ischemic induced cardiomyocyte apoptosis via the PTEN/AKT signaling pathway. Next, the present study further identified the underlying molecular mechanism by which EPI safeguards cardiac functions. As earlier work demonstrated, PTEN is a primary negative regulator of the PI3K/AKT signaling pathway (24). To examine whether PTEN mediates the cardiac protective action of EPI, this study used western blot analysis to compare protein levels of components of the PTEN/AKT pathway in each group. As shown in Fig. 4A, compared with the sham group, myocardial ischemia significantly induced the decrease of $\mathrm{Bcl}-2$ and the increase of Bax and cleaved caspase-3 in MI group, which were rescued by the administration of EPI. However, in the MI+EPI+LY294002 group, LY294002 significantly abolished the rescue effect of EPI. Furthermore, Fig. 4B indicated that PTEN and the $\mathrm{p}-\mathrm{AKT}$ /T-AKT ratio were upregulated and downregulated, respectively, in the MI group compared with the sham group. PTEN and $\mathrm{p}-\mathrm{AKT} / \mathrm{T}-\mathrm{AKT}$ ratio were decreased and increased, respectively, in the MI+EPI group compared with the MI group, which were both significantly reversed by LY294002 co-treatment in the MI+EPI+LY294002 group. Taken together, these results demonstrated that EPI 

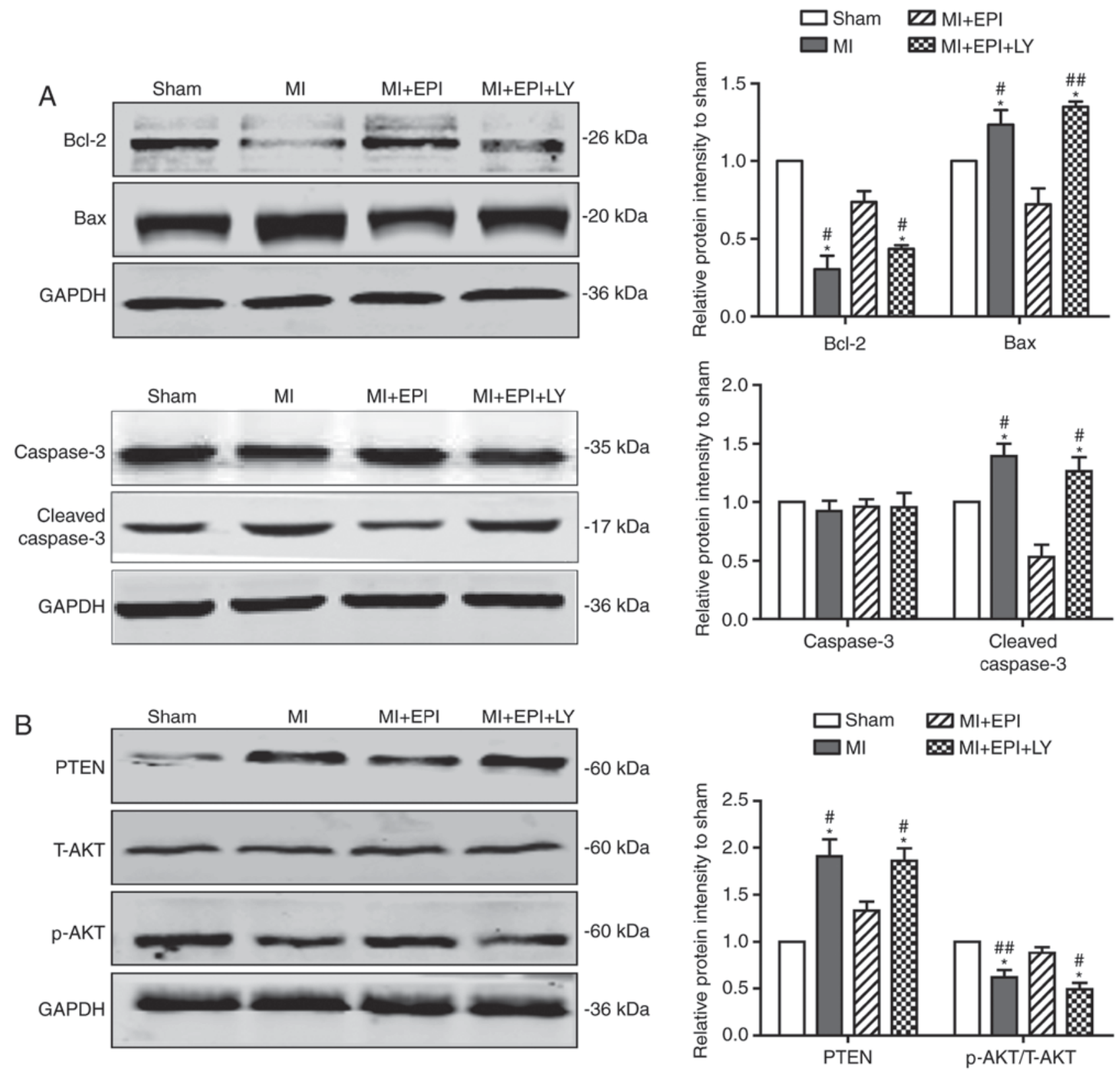

Figure 4. EPI inhibits myocardial ischemia-induced cardiomyocyte apoptosis, which is reversed by LY294002. (A) Western blot analysis for Bcl-2, Bax, caspase-3 and cleaved caspase-3 in mouse hearts from sham group, MI group, MI+EPI group and MI+EPI+LY294002 group. EPI upregulated the expression of Bcl-2 and downregulated the expression of Bax-3 and cleaved caspase-3 in ischemic hearts, which were reversed by LY294002. (B) Western blot analysis for PTEN, T-AKT and p-AKT. EPI upregulated the expression ratio of p-AKT/T-AKT and downregulated the expression of PTEN in ischemic hearts, which were reversed by LY294002. Data are expressed as the mean \pm standard error $(n=6)$. ${ }^{*} \mathrm{P}<0.05$ and ${ }^{* *} \mathrm{P}<0.01$ vs. sham; ${ }^{*} \mathrm{P}<0.05$ and ${ }^{\# \#} \mathrm{P}<0.01$ vs. MI+EPI. MI, myocardial ischemia; EPI, (-)-epicatechin; LY, LY294002; Bcl-2, B-cell lymphoma-2; Bax, B-cell lymphoma-2-associated X protein; PTEN, phosphatase and tensin homolog; T-AKT, total protein kinase B; p-AKT, phosphorylated protein kinase B.

exerts an anti-apoptotic function during myocardial ischemia in a PTEN/AKT pathway dependent manner.

EPI protects cardiomyocytes from hypoxia-induced apoptosis via the PTEN/AKT signaling pathway. The above results indicated that EPI protects myocardial ischemia-induced heart injury through the PTEN/AKT signaling pathway in vivo. To further confirm the effect of EPI on cardiomyocytes, NMCMs were incubated under normal or hypoxic condition in vitro. As shown in Fig. 5A, cell viability was decreased in ANO group when compared with the control group. Treatment with EPI rescued the decrease of cell viability induced by hypoxia, which was significantly counteracted by LY294002 co-treatment (Fig. 5A). In agreement with the previous results in vivo, hypoxia induced the decrease of $\mathrm{Bcl}-2$ and the increase of $\mathrm{Bax}$ and cleaved caspase- 3 in the NMCMs of ANO group in vitro. EPI treatment significantly rescued the decrease of Bcl-2 and suppressed the increase of Bax and cleaved caspase- 3 in hypoxia-treated NMCMs when compared to the level of the control group, which was abolished by the addition of LY294002 (Fig. 5B). In addition, treatment with EPI significantly attenuated hypoxia-induced upregulation of PTEN and downregulation of p-AKT when compared with the MI group. Similarly, LY294002 treatment reversed the effect of 

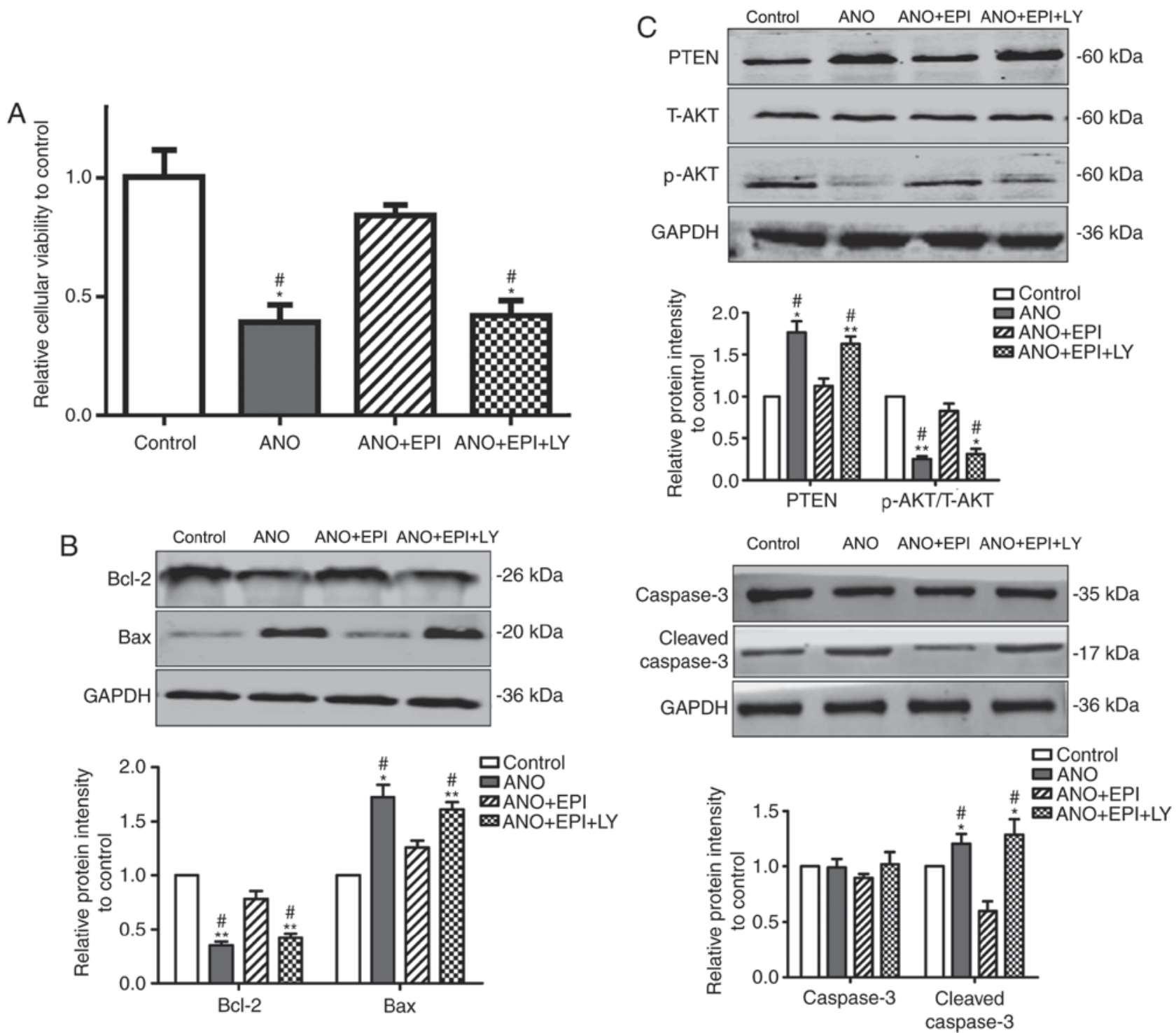

Figure 5. EPI protects cardiomyocytes from hypoxia-induced apoptosis, which is reversed by LY294002. (A) Cell viability was detected by MTT. EPI increased cardiomyocyte viability under hypoxia culture conditions, whereas LY294002 reversed this effect of EPI. (B) Western blot analysis for Bcl-2, Bax, caspase-3 and cleaved caspase-3 in cardiomyocytes. EPI upregulated the expression of Bcl-2 and downregulated the expression levels of Bax-3 and cleaved caspase-3 in cardiomyocytes cultured under hypoxia conditions, which were reversed by LY294002. (C) Western blot analysis for PTEN, T-AKT and p-AKT. EPI upregulated the expression ratio of P-AKT/T-AKT and downregulated the expression of PTEN in cardiomyocytes cultured under anoxia condition, which were reversed by LY294002. Data are expressed as the mean \pm standard error $(\mathrm{n}=6)$. ${ }^{*} \mathrm{P}<0.05$ and ${ }^{* * *} \mathrm{P}<0.01$ vs. control; ${ }^{*} \mathrm{P}<0.05$ vs. ANO+EPI. ANO, anoxia; EPI, (-)-epicatechin; LY, LY294002; Bcl-2, B-cell lymphoma-2; Bax, B-cell lymphoma-2-associated X protein; PTEN, phosphatase and tensin homolog; T-AKT, total protein kinase B; p-AKT, phosphorylated protein kinase B.

EPI on the PTEN and the p-AKT. The level of T-AKT was not affected in each group (Fig. 5C). Taken together, these results indicated that EPI protects cardiomyocytes from hypoxia-induced apoptosis through the PTEN/AKT pathway in vitro.

\section{Discussion}

The present study demonstrated that EPI serves a significant role in protecting against cardiac ischemia in vivo and in vitro. In addition, the results revealed that the cardiac protective effect of EPI was derived from its anti-apoptotic ability and these effects were mediated through the AKT-PTEN signaling pathway.

Myocardial ischemia is one of primary causes of sudden death worldwide (25). Although life science and medicine are improving, there is still no effective method of prevention and treatment for myocardial ischemia injury. Therefore, it is necessary to explore novel therapeutics for this disease. For the past decade, microRNAs and long non-coding (lnc)RNAs have been confirmed to exert significant regulatory effect of myocardial ischemia (26). However, due to the limitation of drug-delivery methods to expediently transport therapeutic microRNAs or IncRNAs into patients' cardiomyocytes, therapy of myocardial ischemia with microRNAs and lncRNAs are not effective and widely used in clinical treatments. Therefore, exploring a natural substance that may be easily applied is required for treating myocardial ischemia.

The present study investigated the effects of EPI on cardiac ischemia injury. It was observed that EPI suppressed mouse 
myocardial hypertrophy and fibrosis induced by myocardial ischemia in vivo. EPI is a common substance which is contained in numerous daily foods and drinks. Therefore, it is worthy to explore the anti-myocardial ischemia effects of EPI. Due to its antioxidative effect, EPI has been demonstrated to inhibit cancer, diabetes mellitus and insulin resistance (27-29). In addition, EPI was predicted to decrease the possibility of developing cardiovascular disease by increasing flow-mediated dilation of endothelium and decreasing platelet aggregation $(30,31)$. Certain studies have demonstrated that EPI is able to alleviate cardiomyocyte hypertrophy $(11,32,33)$, which is consistent with the results of the present study. The protective effect of EPI on cardiomyocyte hypertrophy was shown to occur via a reduction in oxidative stress and an improvement in mitochondrial structure and function $(8,9)$. The present study revealed that EPI significantly decreased myocardial ischemia-induced apoptosis of cardiomyocytes and increased levels of cleaved caspase- 3 and Bax which are considered to be apoptotic molecular markers. Taken together, the in vivo results indicated that EPI may prevent myocardial ischemia-induced cardiac injury through inhibiting hypertrophy, fibrosis and apoptosis. This hypothesis was further confirmed on NMCMs which were cultured under hypoxic conditions to stimulate myocardial ischemia in vitro.

Dysregulation of multiple signaling pathways has been shown to be implicated in myocardial ischemia (34). As a primary intracellular signaling pathway, the PI3K/AKT pathway modulates cell apoptosis, proliferation, differentiation and autophagy under physiological and pathological conditions. AKT serves an important role in suppressing cellular apoptosis. It is able to decrease pro-apoptotic Bad and Bax levels, but elevates anti-apoptotic Bcl-2 (35). Conversely, it diminishes the release of p53, which promotes apoptosis in the presence of DNA damage or other stress (36). Therefore, the overall effect of the PI3K/AKT signaling pathway is to promote cellular survival and to reduce apoptosis. As a major negative regulator of the $\mathrm{PI} 3 \mathrm{~K} / \mathrm{AKT}$ signaling pathway, PTEN is a key molecule in the development of many cardiovascular diseases especially in myocardial ischemia. This study demonstrated that the administration of EPI suppressed the increase of PTEN induced by myocardial ischemia and thus rescued the decrease of AKT signaling. Therefore, it should be noted that the regulatory role of EPI on the PTEN/AKT signaling pathway may at least in part to inhibit cardiomyocyte apoptosis induced by myocardial ischemia.

In conclusion, the research of the present study demonstrated the protective effect of EPI on mouse myocardial ischemia in vivo and in vitro. The present work revealed EPI as a novel anti-apoptotic reagent that attenuates ischemic cardiac injury through targeting the PTEN/AKT signaling pathway. These results indicated that EPI may be important for the development of novel therapeutic strategies for protecting against ischemic insults. Therefore, EPI may represent a novel approach for cardiac ischemia disease prevention and treatment.

\section{Acknowledgements}

Not applicable.

\section{Funding}

No funding was received.

\section{Availability of data and materials}

The datasets used and/or analyzed during the current study are available from the corresponding author on reasonable request.

\section{Authors' contributions}

JL: Study concept and design, acquisition of data, drafting of the manuscript, and statistical analysis; XW: Study concept and design, analysis and interpretation of data, drafting of the manuscript, and statistical analysis; XZ: Acquisition of data, analysis and interpretation of data, and technical support; LG and LW: Acquisition of data, and technical support; XY: Study concept and design, critical revision of the manuscript for important intellectual content, obtained funding, administrative, and study supervision.

\section{Ethics approval and consent to participate}

All experimental procedures were performed in accordance with the Guidelines for the Care and Use of Laboratory Animals set by the US National Institutes of Health (Bethesda, MD, USA), which was approved by the Institutional Animal Care and Use Committee of the Harbin Medical University.

\section{Consent for publication}

Not applicable.

\section{Competing interests}

The authors declare that they have no competing interests.

\section{References}

1. Steg PG, Greenlaw N, Tendera M, Tardif JC, Ferrari R, Al-Zaibag M, Dorian P, Hu D, Shalnova S, Sokn FJ, et al: Prevalence of anginal symptoms and myocardial ischemia and their effect on clinical outcomes in outpatients with stable coronary artery disease: Data from the international observational CLARIFY registry. JAMA Intern Med 174: 1651-1659, 2014.

2. Cheng D, Zhu C, Cao J and Jiang W: The protective effects of polyphenols from jujube peel (Ziziphus Jujube Mill) on isoproterenol-induced myocardial ischemia and aluminum-induced oxidative damage in rats. Food Chem Toxicol 50: 1302-1308, 2012.

3. Misumida N, Kobayashi A, Saeed M, Fox JT and Kanei Y: Electrocardiographic left ventricular hypertrophy as a predictor for nonsignificant coronary artery disease in patients with non-ST-segment elevation myocardial infarction. Angiology 67: 27-33, 2016.

4. Sung HK, Chan YK, Han M, Jahng JWS, Song E, Danielson E, Berger T, Mak TW and Sweeney G: Lipocalin-2 (NGAL) attenuates autophagy to exacerbate cardiac apoptosis induced by myocardial ischemia. J Cell Physiol 232: 2125-2134, 2017.

5. Panza JA, Holly TA, Asch FM, She L, Pellikka PA, Velazquez EJ, Lee KL, Borges-Neto S, Farsky PS, Jones RH, et al: Inducible myocardial ischemia and outcomes in patients with coronary artery disease and left ventricular dysfunction. J Am Coll Cardiol 61: 1860-1870, 2013. 
6. Patel RD and Saver JL: Evolution of reperfusion therapies for acute brain and acute myocardial ischemia: A systematic, comparative analysis. Stroke 44: 94-98, 2013.

7. Shay J, Elbaz HA, Lee I, Zielske SP, Malek MH and Huttemann M: Molecular mechanisms and therapeutic effects of (-)-epicatechin and other polyphenols in cancer, inflammation, diabetes, and neurodegeneration. Oxidat Med Cell Longev 2015: 181260, 2015.

8. Quine SD and Raghu PS: Effects of (-)-epicatechin, a flavonoid on lipid peroxidation and antioxidants in streptozotocin-induced diabetic liver, kidney and heart. Pharmacol Rep 57: 610-615, 2005.

9. Yamazaki KG, Romero-Perez D, Barraza-Hidalgo M, Cruz M, Rivas M, Cortez-Gomez B, Ceballos G and Villarreal F: Short- and long-term effects of (-)-epicatechin on myocardial ischemia-reperfusion injury. Am J Physiol Heart Circulat Physiol 295: H761-H767, 2008.

10. Yamazaki KG, Taub PR, Barraza-Hidalgo M, Rivas MM Zambon AC, Ceballos G and Villarreal FJ: Effects of (-)-epicatechin on myocardial infarct size and left ventricular remodeling after permanent coronary occlusion. J Am Coll Cardiol 55: 2869-2876, 2010

11. Sheng R, Gu ZL and Xie ML: Epigallocatechin gallate, the major component of polyphenols in green tea, inhibits telomere attrition mediated cardiomyocyte apoptosis in cardiac hypertrophy. Int J Cardiol 162: 199-209, 2013.

12. Suzuki J, Ogawa M, Maejima Y, Isobe K, Tanaka H, Sagesaka YM and Isobe M: Tea catechins attenuate chronic ventricular remodeling after myocardial ischemia in rats. J Mol Cell Cardiol 42 432-440, 2007.

13. Ke Z, Wang G, Yang L, Qiu H, Wu H, Du M, Chen J, Song J, Jia X and Feng L: Crude terpene glycoside component from Radix paeoniae rubra protects against isoproterenol-induced myocardial ischemic injury via activation of the PI3K/AKT/mTOR signaling pathway. J Ethnopharmacol 206: 160-169, 2017.

14. Cui G, Shan L, Hung M, Lei S, Choi I, Zhang Z, Yu P, Hoi P, Wang Y and Lee SM: A novel Danshensu derivative confers cardioprotection via PI3K/Akt and Nrf2 pathways. Int J Cardiol 168: 1349-1359, 2013.

15. Zhang J, Yu XH, Yan YG, Wang C and Wang WJ: PI3K/Akt signaling in osteosarcoma. Clin Chim Acta 444: 182-192, 2015.

16. Ouyang ZH, Wang WJ, Yan YG, Wang B and Lv GH: The PI3K/Akt pathway: A critical player in intervertebral disc degeneration. Oncotarget 8: 57870-57881, 2017.

17. De Los Santos S, García-Pérez V, Hernández-Reséndiz S Palma-Flores C, González-Gutiérrez CJ, Zazueta C, Canto P and Coral-Vázquez RM: (-)-Epicatechin induces physiological cardiac growth by activation of the PI3K/Akt pathway in mice. Mol Nutri Food Res 61, 2017.

18. Li C, Li X, Gao X, Zhang R, Zhang Y, Liang H, Xu C, Du W, Zhang Y, Liu X, et al: MicroRNA-328 as a regulator of cardiac hypertrophy. Int J Cardiol 173: 268-276, 2014.

19. Pan Z, Sun X, Shan H, Wang N, Wang J, Ren J, Feng S, Xie L, Lu C, Yuan Y, et al: MicroRNA-101 inhibited postinfarct cardiac fibrosis and improved left ventricular compliance via the FBJ osteosarcoma oncogene/transforming growth factor- $\beta 1$ pathway. Circulation 126: 840-850, 2012.

20. Abbate A, Salloum FN, Van Tassell BW, Vecile E, Toldo S, Seropian I, Mezzaroma E and Dobrina A: Alterations in the interleukin-1/interleukin-1 receptor antagonist balance modulate cardiac remodeling following myocardial infarction in the mouse. PLoS One 6: e27923, 2011.

21. Panneerselvam M, Tsutsumi YM, Bonds JA, Horikawa YT, Saldana M, Dalton ND, Head BP, Patel PM, Roth DM and Patel HH: Dark chocolate receptors: Epicatechin-induced cardiac protection is dependent on delta-opioid receptor stimulation. Am J Physiol Heart Circ Physiol 299: H1604-H1609, 2010.
22. Wu AZ, Loh SH, Cheng TH, Lu HH and Lin CI: Antiarrhythmic effects of (-)-epicatechin-3-gallate, a novel sodium channel agonist in cultured neonatal rat ventricular myocytes. Biochem Pharmacol 85: 69-80, 2013

23. Feng W, Hwang HS, Kryshtal DO, Yang T, Padilla IT, Tiwary AK, Puschner B, Pessah IN and Knollmann BC: Coordinated regulation of murine cardiomyocyte contractility by nanomolar (-)-epigallocatechin-3-gallate, the major green tea catechin. Mol Pharmacol 82: 993-1000, 2012.

24. Siddall HK, Warrell CE, Yellon DM and Mocanu MM: Ischemia-reperfusion injury and cardioprotection: Investigating PTEN, the phosphatase that negatively regulates PI3K, using a congenital model of PTEN haploinsufficiency. Basic Res Cardiol 103: 560-568, 2008

25. Ounzain S, Crippa S and Pedrazzini T: Small and long non-coding RNAs in cardiac homeostasis and regeneration. Biochim Biophys Acta 1833: 923-933, 2013

26. Wang JX, Zhang XJ, Li Q, Wang K, Wang Y, Jiao JQ, Feng C, Teng S, Zhou LY, Gong Y, et al: MicroRNA-103/107 regulate programmed necrosis and myocardial ischemia/reperfusion injury through targeting FADD. Circ Res 117: 352-363, 2015.

27. Rein D, Lotito S, Holt RR, Keen CL, Schmitz HH and Fraga CG: Epicatechin in human plasma: In vivo determination and effect of chocolate consumption on plasma oxidation status. J Nutri 130 (8S Suppl): S2109-S2114, 2000.

28. Del Rio D, Rodriguez-Mateos A, Spencer JP, Tognolini M, Borges G and Crozier A: Dietary (poly)phenolics in human health: Structures, bioavailability, and evidence of protective effects against chronic diseases. Antioxidants Redox Signal 18 1818-1892, 2013

29. Cremonini E, Bettaieb A, Haj FG, Fraga CG and Oteiza PI: (-)-Epicatechin improves insulin sensitivity in high fat diet-fed mice. Arch Biochem Biophys 599: 13-21, 2016.

30. Drouin A, Bolduc V, Thorin-Trescases N, Bélanger É, Fernandes P, Baraghis E, Lesage F, Gillis MA, Villeneuve L, Hamel E, et al: Catechin treatment improves cerebrovascular flow-mediated dilation and learning abilities in atherosclerotic mice. Am J Physiol Heart Circ Physiol 300: H1032-H1043, 2011.

31. Lotito SB and Frei B: Consumption of flavonoid-rich foods and increased plasma antioxidant capacity in humans: Cause, consequence, or epiphenomenon? Free Radic Biol Med 41: 1727-1746, 2006.

32. Chen DD, Dong YG, Liu D and He JG: Epigallocatechin-3-gallate attenuates cardiac hypertrophy in hypertensive rats in part by modulation of mitogen-activated protein kinase signals. Clin Exp Pharmacol Physiol 36: 925-932, 2009.

33. Hao J, Kim CH, Ha TS and Ahn HY: Epigallocatechin-3 gallate prevents cardiac hypertrophy induced by pressure overload in rats. J Vet Sci 8: 121-129, 2007.

34. Lau E: Complex disease: Piecing together the puzzle of coronary artery disease. Nat Rev Genet 15: 572-573, 2014.

35. Atif F, Yousuf S and Stein DG: Anti-tumor effects of progesterone in human glioblastoma multiforme: Role of PI3K/Akt/mTOR signaling. J Steroid Biochem Mol Biol 146: 62-73, 2015.

36. Ji H, Ding Z, Hawke D, Xing D, Jiang BH, Mills GB and Lu Z: AKT-dependent phosphorylation of Niban regulates nucleophosmin- and MDM2-mediated p53 stability and cell apoptosis. EMBO Rep 13: 554-560, 2012.

This work is licensed under a Creative Common

Attribution-NonCommercial-NoDerivatives 4.0 International (CC BY-NC-ND 4.0) License. 